\title{
Three-dimensional Charge Density and Electric Field Mapping of an Electrically Biased Needle Using Off-axis Electron Holography
}

Fengshan Zheng ${ }^{1}$, Vadim Migunov ${ }^{1}$, Jan Caron ${ }^{1}$, Hongchu Du ${ }^{1}$, Giulio Pozzi ${ }^{2}$ and Rafal DuninBorkowski $^{1}$

${ }^{1}$ Forschungszentrum Juelich, Juelich, Nordrhein-Westfalen, Germany, ${ }^{3}$ Università di Bologna, Bologna, Emilia-Romagna, Italy

The electrical properties of nanoscale electronic devices, such as $p$ - $n$ junctions, transistors and lightemitting diodes, are affected strongly by the presence of defects, dopants, interfaces and surfaces [1]. Just as the performance of field emitters is influenced by geometrical factors such as their shape, curvature and crystallographic orientation [2], in atom probe tomography the shape, chemistry and defects in a needleshaped specimen affect the trajectories of field-evaporated ions and the fidelity of the reconstruction of the three-dimensional (3D) atomic positions [3], while tip-enhanced catalytic efficiency is influenced by the shape and species of the tip [4]. A knowledge of the 3D charge density and electric field inside and around such nanostructures is therefore required.

Off-axis electron holography provides direct access to the projected electrostatic potential within and around a specimen in the transmission electron microscope (TEM) [5]. Here, we combine off-axis electron holography with electron tomography and a model-based iterative reconstruction algorithm [6] to determine the 3D charge density, electric field and potential within and around an electrically-biased C fibre needle. The needle was prepared using a standard focused ion beam (FIB) preparation procedure. A Nanofactory holder was used for electrical biasing. A $\mu \mathrm{m}$-sized Au tip was used as a counter-electrode at a distance of $4.5 \mu \mathrm{m}$ from the needle. The diameter of the apex of the needle was $\sim 60 \mathrm{~nm}$ and its length was $\sim 2.5 \mu \mathrm{m}$. Two tomographic tilt series of holographic phase images were acquired between $-52^{\circ}$ and $+48^{\circ}$ with a tilt increment of $4^{\circ}$. One series was acquired without an electrical bias applied to the needle. This dataset includes only the mean inner potential (MIP) contribution to the phase. It was used to reconstruct the 3D morphology of the needle and to subtract the MIP contribution to the phase from a second dataset recorded with an electrical bias of $40 \mathrm{~V}$ applied to the needle.

Figure 1a shows a visualisation of the reconstructed 3D charge density in the needle. The charge density is greatest at the apex and has a maximum value of $2.94 \times 10^{18} \mathrm{~cm}^{-3}$. Selected 2D slices extracted from the $3 \mathrm{D}$ reconstruction are shown in Figs 1(b-f). The $x y$ plane of the charge density (Fig. 1b) reveals an asymmetry (white arrow " 1 "), which is absent in the $y z$ plane (Fig. 1c). The fact that the majority of the charge is located close to the surface of the needle is visible in the yzplane (Fig. 1c). Another region with a locally increased charge density, which is marked by red dashed ellipses in Figs 1(b, c), may be associated with damage by Ga bombardment during sample preparation. Figure 2 shows a combined plot of the electric field and electrostatic potential in the central $x y$ plane calculated from the reconstructed 3D charge density on the assumption that image charges are defined by the counter-electrode. The strength of the electric field is greatest close to the apex of the needle and has a maximum value of $\sim 0.25 \mathrm{GV} / \mathrm{m}[7]$. 

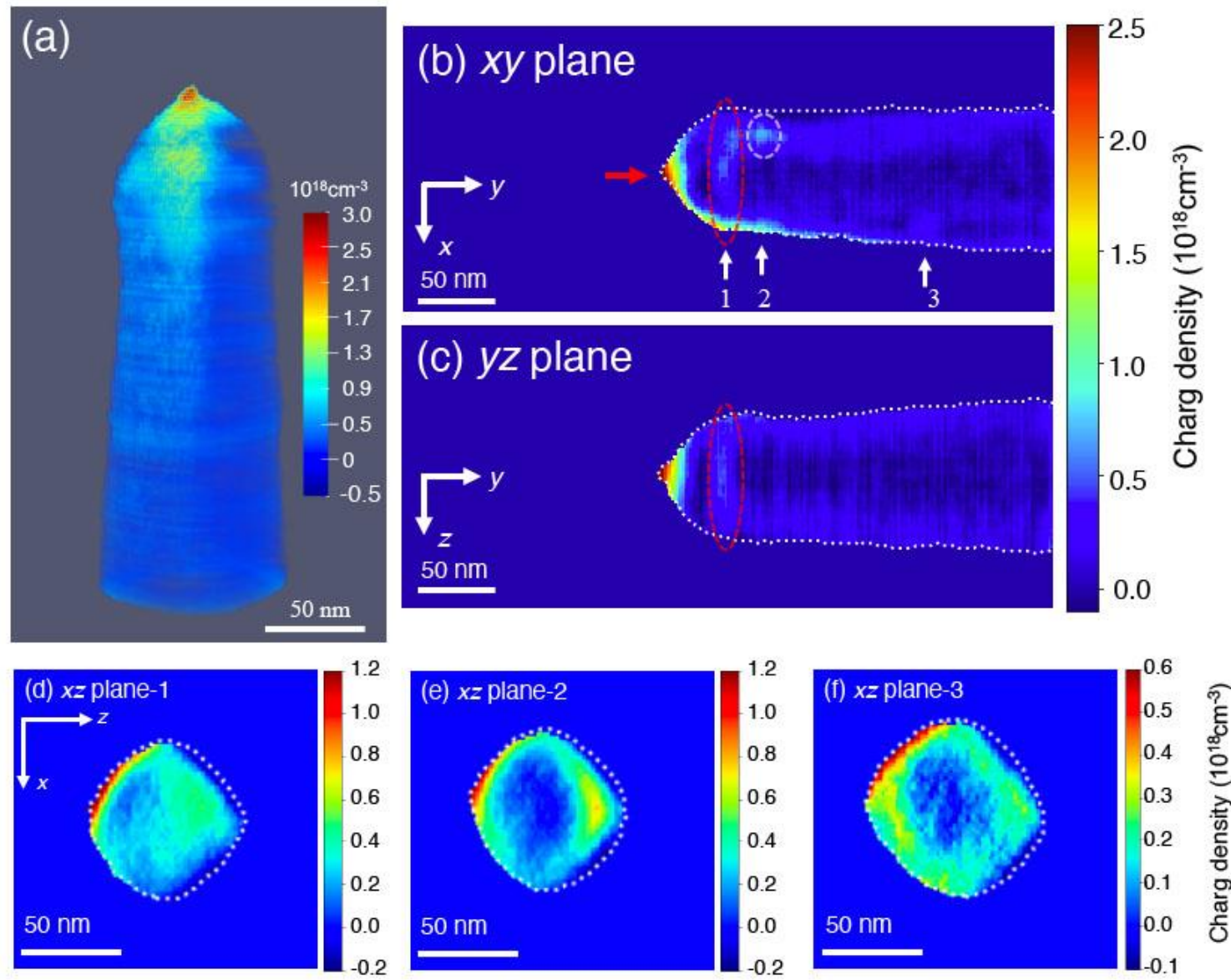

Figure 1. 3D charge density in an electrically biased $\mathrm{C}$ fibre needle reconstructed using electron holographic tomography and model-based iterative reconstruction. The needle is biased at $+40 \mathrm{~V}$ with a distance of $4.5 \mu \mathrm{m}$ to a $\mathrm{Au}$ counter-electrode.(a) Side view of the 3D charge density. (b, c) 2D slices extracted from the reconstructed 3D charge density in the xy and yz planes, respectively. (d-f) Three representative 2D slices of charge density in the xz plane extracted from the 3D charge density. The planes are marked in (b). The white dotted lines in (b-f) mark the edge of the needle. 


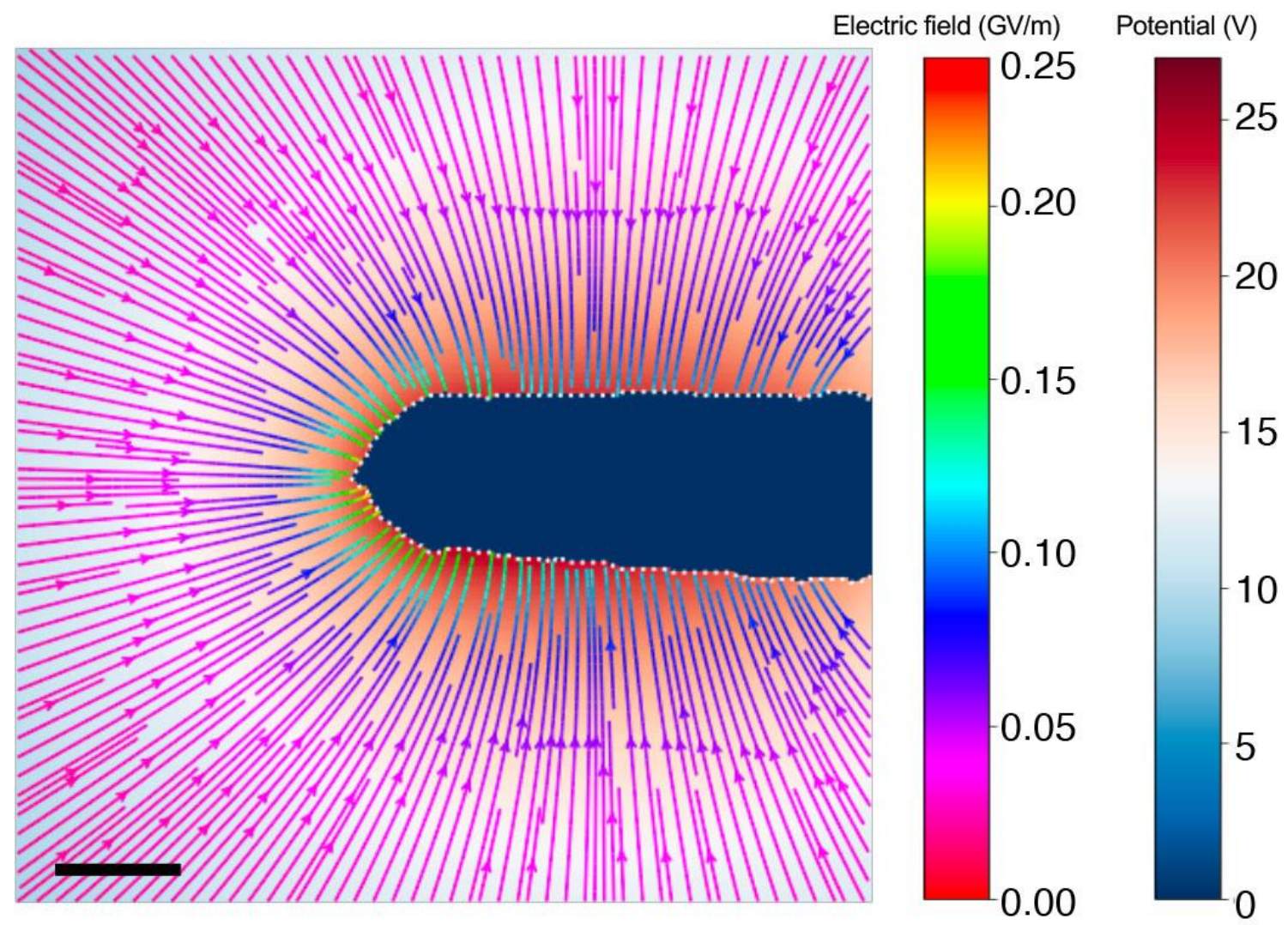

Figure 2. Combination of a streamline plot of electric field and a colour map of electrostatic potential in the central $x y$ section of the $\mathrm{C}$ fibre needle determined from the reconstructed $3 \mathrm{D}$ charge density distribution shown in Fig. 1. The interior of the needle is marked in blue. The scale bar is $50 \mathrm{~nm}$.

\section{References}

[1] S.M. Sze, Semiconductor devices: physics and technology, Wiley, 2008.

[2] H. Zhang, et.al., Nat. Nanotech. 11 (2016) 273.

[3] F. Vurpillot, et.al., Ultramicroscopy. 159 (2015) 202.

[4] Y. Su, et.al., Nat. Nanotech. 11 (2016) 609.

[5] H. Lichte, et.al., Rep. Prog. Phys. 71 (2007) 016102.

[6] F. Zheng, et.al, J. Elec. Spec. Rel. Phenom. 2020, in press.

[7] The authors acknowledge the European Union for funding through the Marie Curie Initial Training Network Grant No. 606988 under FP7-PEOPLE-2013-ITN). 